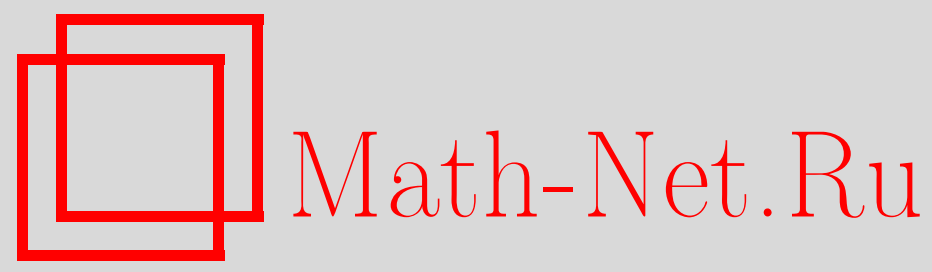

В. Н. Русак, И. В. Рыбаченко, Свойства функций и приближение сумматорными рациональными операторами на действительной оси, Матем. заметки, 2004, том 76, выпуск 1, 111-118

DOI: https://doi.org/10.4213/mzm93

Использование Общероссийского математического портала Math-Net.Ru подразумевает, что вы прочитали и согласны с пользовательским соглашением http://www . mathnet.ru/rus/agreement

Параметры загрузки:

IP : 3.85 .73 .92

26 апреля 2023 г., 18:04:06 


\section{СВОЙСТВА ФУНКЦИЙ И ПРИБЛИЖЕНИЕ СУММАТОРНЫМИ РАЦИОНАЛЬНЫМИ ОПЕРАТОРАМИ НА ДЕЙСТВИТЕЛЬНОЙ ОСИ}

\section{В. Н. Русак, И. В. Рыбаченко}

В пространстве $C(\mathbb{R})$ построены специальные сумматорные операторы типа Джексона с рациональными ядрами и при подходящем выборе полюсов найдены порядковые оценки для уклонений этих операторов от функций из определенных классов.

Библиография: 10 названий.

Интегральные операторы с рациональными ядрами нашли применение как в рациональных приближениях с фиксированными полюсами, так и в наилучших рациональных аптроксимациях со свободными полюсами на функциональных классах [1]-[4]. Первые исследования по интерполяционным и сумматорным рациональным операторам в пространстве $C(\mathbb{R})$ непрерьвных на действительной оси функций были вьполнены Е. А. Ровбой [5], [6]. В данной работе предложена иная по сравнению с [6] конструкция сумматорных рациональных операторов джексоновского типа и при подходящем выборе полюсов найдены порядковые оценки для их уклонений от функций из определенных классов.

По заданной системе $\left\{z_{k}\right\}_{k=1}^{n}, \operatorname{Im} z_{k}>0$, введем синус-дробь Бернштейна $N_{2 n}(x)$, полагая

$$
N_{2 n}(x)=\sin 2 \Phi_{n}(x), \quad \Phi_{n}(x)=\sum_{k=1}^{n} \arg \left(z_{k}-x\right) .
$$

Известно, что $N_{2 n}(x)$ - рациональная функция порядка $2 n$ с полюсами в точках $z_{k}$ и $\bar{z}_{k}, k=1, \ldots, n$, и с нулями $[1$, с. 14$]$

$$
\left\{x_{k}\right\}_{k=1}^{2 n}, \quad x_{1}<x_{2}<\cdots<x_{2 n-1}, \quad x_{2 n}=\infty .
$$

Заметим также, что из (1) вытекает

$$
\Phi_{n}^{\prime}(x)=\sum_{k=1}^{n} \frac{\beta_{k}}{\left(\alpha_{k}-x\right)^{2}+\beta_{k}^{2}}, \quad z_{k}=\alpha_{k}+i \beta_{k} .
$$

Полагаем, что

$$
\sum_{k=1}^{2 n-1} \frac{\sin ^{4}\left[\Phi_{n}(x)-\Phi_{n}\left(x_{k}\right)\right]}{\left(x-x_{k}\right)^{4} \Phi_{n}^{\prime}\left(x_{k}\right)} \stackrel{\text { def }}{=} 2 g_{n}(x)
$$

Работа выполнена при финансовой поддержке Белорусского республиканского фонда фундаментальных исследований, грант № $\$ 02 \mathrm{P}-057$. 
и для функции $f \in C(\mathbb{R})$ определяем сумматорный рациональный оператор посредством равенства

$$
D_{4 n-4}(x, f) \stackrel{\text { def }}{=} \frac{1}{2 g_{n}(x)} \sum_{k=1}^{2 n-1} f\left(x_{k}\right) \frac{\sin ^{4}\left[\Phi_{n}(x)-\Phi_{n}\left(x_{k}\right)\right]}{\left(x-x_{k}\right)^{4} \Phi_{n}^{\prime}\left(x_{k}\right)} .
$$

Непосредственно из определения ясно, что $D_{4 n-4}-$ положительный оператор, точньй на константах, и его значение $D_{4 n-4}(x, f)$ - рациональная функция порядка $4 n-4$.

ЛЕМма 1. Справедливо равенство

$$
g_{n}(x)=\frac{2}{3}\left[\Phi_{n}^{\prime}(x)\right]^{3}+\frac{1}{6} \sum_{k=1}^{n} \beta_{k} \frac{3\left(\alpha_{k}-x\right)^{2}-\beta_{k}^{2}}{\left(\left(\alpha_{k}-x\right)^{2}+\beta_{k}^{2}\right)^{3}} .
$$

ДокАЗАтЕЛЬСтво. Рассмотрим контурньй интеграл

$$
J_{n}=\frac{1}{2 \pi i} \int_{\gamma} R_{n}(t) d t \stackrel{\text { def }}{=} \frac{1}{2 \pi i} \int_{\gamma} \frac{\sin ^{4}\left[\Phi_{n}(x)-\Phi_{n}(t)\right]}{(x-t)^{4}} \operatorname{ctg} 2 \Phi_{n}(t) d t,
$$

где $\gamma$ - замкнутый кусочно-гладкий контур, окружающий точки $\left\{z_{k}, \bar{z}_{k}\right\}_{k=1}^{n}$; нули $\left\{x_{j}\right\}_{j=1}^{2 n}$ синус-дроби $N_{2 n}(x)$ находятся во внешности $\gamma$ и интегрирование ведется в положительном направлении относительно внешней области. По теореме о вычетах для внешней области будем иметь с учетом (4)

$$
\begin{aligned}
J_{n} & =\sum_{k=1}^{2 n-1} \operatorname{Res}_{t=x_{k}} R_{n}(t)=\sum_{k=1}^{2 n-1} \lim _{t \rightarrow x_{k}} \frac{\sin ^{4}\left[\Phi_{n}(x)-\Phi_{n}(t)\right]}{(x-t)^{4}} \cos 2 \Phi_{n}(t) \frac{t-x_{k}}{\sin 2 \Phi_{n}(t)} \\
& =\sum_{k=1}^{2 n-1} \frac{\sin ^{4}\left[\Phi_{n}(x)-\Phi_{n}\left(x_{k}\right)\right]}{\left(x-x_{k}\right)^{4}} \frac{1}{2 \Phi_{n}^{\prime}\left(x_{k}\right)}=g_{n}(x),
\end{aligned}
$$

поскольку $R_{n}(t)$ имеет полюсы 1-го порядка в точках $\left\{x_{k}\right\}_{k=1}^{2 n-1}$ и нуль третьего порядка в бесконечно удаленной точке. С другой стороны, применяя теорему о вычетах для внутренней области, получим, считая для определенности все параметры $\left\{z_{k}\right\}_{k=1}^{n}$ различными:

$$
J_{n}=-\sum_{k=1}^{n}\left(\operatorname{Res}_{t=z_{k}} R_{n}(t)+\operatorname{Res}_{t=\bar{z}_{k}} R_{n}(t)\right) .
$$

С учетом (1) и формул Эйлера (см. также $[1$, c. 116]) подьнтегральная функция из (7) может быть записана в виде

$$
R_{n}(t)=\frac{1}{16(t-x)^{4}} \frac{\left(a_{n}(x) b_{n}(t)-a_{n}(t) b_{n}(x)\right)^{4}}{a_{n}^{2}(x) a_{n}^{2}(t) b_{n}^{2}(x) b_{n}^{2}(t)} i \frac{a_{n}^{2}(t)+b_{n}^{2}(t)}{a_{n}^{2}(t)-b_{n}^{2}(t)},
$$

где

$$
a_{n}(t)=\prod_{k=1}^{n}\left(t-z_{k}\right), \quad b_{n}(t)=\prod_{k=1}^{n}\left(t-\bar{z}_{k}\right)
$$

и, следовательно, $R_{n}(t)$ имеет полюсы второй кратности в точках $\left\{z_{k}\right\}_{k=1}^{n}$ и $\left\{\bar{z}_{k}\right\}_{k=1}^{n}$, если все параметры $\left\{z_{k}\right\}_{k=1}^{n}$ различные. 
Вводя еще обозначение

$$
G_{n}(x, t) \stackrel{\text { def }}{=} \frac{\sin ^{4}\left[\Phi_{n}(x)-\Phi_{n}(t)\right]}{(x-t)^{4}}
$$

будем иметь

$$
\begin{aligned}
\operatorname{Res}_{t=z_{k}} R_{n}(t) & =\operatorname{Res}_{t=z_{k}} G_{n}(x, t) \operatorname{ctg} 2 \Phi_{n}(t)=\lim _{t \rightarrow z_{k}} \frac{\partial}{\partial t}\left(G_{n}(x, t) \operatorname{ctg} 2 \Phi_{n}(t)\left(t-z_{k}\right)^{2}\right) \\
& =\lim _{t \rightarrow z_{k}}\left[\frac{\partial}{\partial t}\left(G_{n}(x, t)\left(t-z_{k}\right)^{2}\right) \operatorname{ctg} 2 \Phi_{n}(t)+G_{n}(x, t)\left(t-z_{k}\right)^{2} \frac{d}{d t} \operatorname{ctg} 2 \Phi_{n}(t)\right] \\
& =\lim _{t \rightarrow z_{k}} \frac{\partial}{\partial t}\left(G_{n}(x, t)\left(t-z_{k}\right)^{2}\right)(-i)=-i \operatorname{Res}_{t=z_{k}} G_{n}(x, t),
\end{aligned}
$$

поскольку

$$
\left.\frac{d}{d t} \operatorname{ctg} 2 \Phi_{n}(t)\right|_{t=z_{k}, \bar{z}_{k}}=0,\left.\quad \operatorname{ctg} 2 \Phi_{n}(t)\right|_{t=z_{k}}=-i,\left.\quad \operatorname{ctg} 2 \Phi_{n}(t)\right|_{t=\bar{z}_{k}}=i .
$$

Аналогично устанавливается, что

$$
\operatorname{Res}_{t=\bar{z}_{k}} R_{n}(t)=i \operatorname{Res}_{t=\bar{z}_{k}} G_{n}(x, t) .
$$

Подставляя (11) и (12) в соотношение (9), найдем (см. (8))

$$
g_{n}(x)=J_{n}=i\left(\sum_{k=1}^{n} \operatorname{Res}_{t=z_{k}} G_{n}(x, t)-\sum_{k=1}^{n} \operatorname{Res}_{t=\bar{z}_{k}} G_{n}(x, t)\right) .
$$

Отправляясь теперь от известного соотношения [1, с. 144]

$$
\frac{1}{\pi} \int_{-\infty}^{\infty} G_{n}(x, t) d t=\frac{2}{3}\left[\Phi_{n}^{\prime}(x)\right]^{3}+\frac{1}{6} \sum_{k=1}^{n} \beta_{k} \frac{3\left(\alpha_{k}-x\right)^{2}-\beta_{k}^{2}}{\left(\left(\alpha_{k}-x\right)^{2}+\beta_{k}^{2}\right)^{3}},
$$

и вычисляя стоящий слева интеграл с помощью вычетов, найдем

$$
\frac{1}{\pi} \int_{-\infty}^{\infty} G_{n}(x, t) d t=i\left(\sum_{k=1}^{n} \operatorname{Res}_{t=z_{k}} G_{n}(x, t)-\sum_{k=1}^{n} \operatorname{Res}_{t=\bar{z}_{k}} G_{n}(x, t)\right) .
$$

Из (13)-(15) вытекает (6), и доказательство леммы 1 завершено.

Лемма 2. Справедливо неравенство

$$
\sum_{k=1}^{2 n-1} \frac{\sin ^{2}\left[\Phi_{n}(x)-\Phi_{n}\left(x_{k}\right)\right]}{\left(x-x_{k}\right)^{2} \Phi_{n}^{\prime}\left(x_{k}\right)} \leqslant 2 \Phi_{n}^{\prime}(x), \quad x \in \mathbb{R} .
$$

Доказательство связано с использованием контурного интеграла

$$
\frac{1}{2 \pi i} \int_{\gamma} \frac{\sin ^{2}\left[\Phi_{n}(x)-\Phi_{n}(t)\right]}{(x-t)^{2}} \operatorname{ctg} 2 \Phi_{n}(t) d t
$$

и соображений, применявшихся при доказательстве предыдушей леммы. Заметим, что на этот раз вычет в бесконечно удаленной точке не будет равен нулю; именно этим объясняется знак неравенства в соотношении (16). 
Лемма 3. Для любого $x \in \mathbb{R}$ справедливы неравенства

$$
g_{n}(x) \geqslant \frac{1}{2}\left[\Phi_{n}^{\prime}(x)\right]^{3}, \quad g_{n}(x) \geqslant \frac{1}{2} \sum_{k=1}^{n} \frac{\beta_{k}}{\left[\left(\alpha_{k}-x\right)^{2}+\beta_{k}^{2}\right]^{2}} .
$$

ДокаЗАТЕЛЬСтво. Действительно, из (6) и (3) имеем

$$
g_{n}(x)>\frac{1}{2}\left[\Phi_{n}^{\prime}(x)\right]^{3}+\frac{1}{6}\left(\left[\Phi_{n}^{\prime}(x)\right]^{3}-\sum_{k=1}^{n} \frac{\beta_{k}^{3}}{\left[\left(\alpha_{k}-x\right)^{2}+\beta_{k}^{2}\right]^{3}}\right)>\frac{1}{2}\left[\Phi_{n}^{\prime}(x)\right]^{3},
$$

и первое из соотношений (17) установлено. С другой стороны, после очевидных преобразований имеем

$$
\begin{aligned}
g_{n}(x) & =\frac{1}{2} \sum_{k=1}^{n} \frac{\beta_{k}}{\left[\left(\alpha_{k}-x\right)^{2}+\beta_{k}^{2}\right]^{2}}+\frac{2}{3}\left[\Phi_{n}^{\prime}(x)\right]^{3}-\frac{2}{3} \sum_{k=1}^{n} \frac{\beta_{k}^{3}}{\left[\left(\alpha_{k}-x\right)^{2}+\beta_{k}^{2}\right]^{3}} \\
& >\frac{1}{2} \sum_{k=1}^{n} \frac{\beta_{k}}{\left[\left(\alpha_{k}-x\right)^{2}+\beta_{k}^{2}\right]^{2}},
\end{aligned}
$$

и второе неравенство (17) также доказано.

Теорема 1. Если $f(x)$ равномерно непрерывна на $\mathbb{R}$, то

$$
\left|f(x)-D_{4 n-4}(x, f)\right| \leqslant(\sqrt{2}+1) \omega\left(\frac{1}{\Phi_{n}^{\prime}(x)}\right),
$$

әде $\omega(\delta)$ - модуль непрерывности функции $f(x)$.

ДоКАЗАТЕльСтво. Учитьвая (5), (6) и свойства модуля непрерывности, для уклонения $\left|f(x)-D_{4 n-4}(x, f)\right|$ найдем

$$
\begin{aligned}
\left|f(x)-D_{4 n-4}(x, f)\right| & \leqslant \frac{1}{2 g_{n}(x)} \sum_{k=1}^{2 n-1}\left|f(x)-f\left(x_{k}\right)\right| \frac{\sin ^{4}\left[\Phi_{n}(x)-\Phi_{n}\left(x_{k}\right)\right]}{\left(x-x_{k}\right)^{4} \Phi_{n}^{\prime}\left(x_{k}\right)} \\
& \leqslant \frac{1}{2 g_{n}(x)} \sum_{k=1}^{2 n-1} \omega\left(\left|x-x_{k}\right|\right) \frac{\sin ^{4}\left[\Phi_{n}(x)-\Phi_{n}\left(x_{k}\right)\right]}{\left(x-x_{k}\right)^{4} \Phi_{n}^{\prime}\left(x_{k}\right)} \\
& \leqslant \omega\left(\frac{1}{\Phi_{n}^{\prime}(x)}\right)\left\{\frac{\Phi_{n}^{\prime}(x)}{2 g_{n}(x)} \sum_{k=1}^{2 n-1}\left|\frac{\sin \left[\Phi_{n}(x)-\Phi_{n}\left(x_{k}\right)\right]}{x-x_{k}}\right|^{3} \frac{1}{\Phi_{n}^{\prime}\left(x_{k}\right)}+1\right\} \\
& =\omega\left(\frac{1}{\Phi_{n}^{\prime}(x)}\right)\left\{S_{1}+1\right\} .
\end{aligned}
$$

Используя неравенство Коши для сумм и леммы 1-3, будем иметь

$$
\begin{aligned}
S_{1} & \leqslant \frac{\Phi_{n}^{\prime}(x)}{2 g_{n}(x)} \sqrt{\sum_{k=1}^{2 n-1} \frac{\sin ^{2}\left[\Phi_{n}(x)-\Phi_{n}\left(x_{k}\right)\right]}{\left(x-x_{k}\right)^{2} \Phi_{n}^{\prime}\left(x_{k}\right)}} \sqrt{\sum_{k=1}^{2 n-1} \frac{\sin ^{4}\left[\Phi_{n}(x)-\Phi_{n}\left(x_{k}\right)\right]}{\left(x-x_{k}\right)^{4} \Phi_{n}^{\prime}\left(x_{k}\right)}} \\
& \leqslant \frac{\Phi_{n}^{\prime}(x)}{2 g_{n}(x)} \sqrt{2 \Phi_{n}^{\prime}(x)} \sqrt{2 g_{n}(x)}=\frac{\left[\Phi_{n}^{\prime}(x)\right]^{3 / 2}}{\sqrt{g_{n}(x)}} \leqslant\left[\Phi_{n}^{\prime}(x)\right]^{3 / 2} \frac{\sqrt{2}}{\left[\Phi_{n}^{\prime}(x)\right]^{3 / 2}}=\sqrt{2} .
\end{aligned}
$$

Отсюда и из неравенства (18) вытекает правильность неравенства, содержашегося в теореме 1.

Назовем функцию $\varphi \in C(\mathbb{R})$ простой, если она абсолютно непрерывна, равна нулю вне некоторого отрезка $[a, b]$, именуемого опорным м и и $\left\|\varphi^{\prime}\right\|_{L_{\infty}(\mathbb{R})} \leqslant(b-a)^{-1}$. 
Лемма 4. Если $\varphi(x)$ - простая функиия с опорным отрезком $[\alpha-\beta, \alpha+\beta]$, то для $x \in \mathbb{R}$ справедлива оченка

$$
\left|\varphi(x)-D_{4 n-4}(x, f)\right| \leqslant \frac{6}{\sqrt[3]{g_{n}(x)}} \frac{\beta}{(x-\alpha)^{2}+\beta^{2}}+\frac{16}{g_{n}(x)} \frac{\beta}{\left[(x-\alpha)^{2}+\beta^{2}\right]^{2}} .
$$

ДОКАЗАТЕЛЬСТво. Пусть

$$
E_{x}=\left\{t:|x-t| \leqslant \frac{1}{\sqrt[3]{g_{n}(x)}}\right\}, \quad C E_{x}=\mathbb{R} \backslash E_{x} .
$$

Тогда с учетом определения простой функции, свойств оператора $D_{4 n-4}$, неравенства Коши и лемм $2-3$ будем иметь

$$
\begin{aligned}
\left|\varphi(x)-D_{4 n-4}(x, f)\right| \leqslant & \frac{1}{2 g_{n}(x)} \sum_{x_{k} \in E_{x}} \frac{1}{2 \beta} \frac{1}{\sqrt[3]{g_{n}(x)}} \frac{\sin ^{4}\left[\Phi_{n}(x)-\Phi_{n}\left(x_{k}\right)\right]}{\left(x-x_{k}\right)^{4} \Phi_{n}^{\prime}\left(x_{k}\right)} \\
& +\frac{1}{2 g_{n}(x)} \sum_{x_{k} \in C E_{x}} \frac{1}{2 \beta} \frac{\left|\sin ^{3}\left[\Phi_{n}(x)-\Phi_{n}\left(x_{k}\right)\right]\right|}{\left|x-x_{k}\right|^{3} \Phi_{n}^{\prime}\left(x_{k}\right)} \\
\leqslant & \frac{1}{2 \beta} \frac{1}{\sqrt[3]{g_{n}(x)}}+\frac{1}{2 \beta} \frac{1}{2 g_{n}(x)} \sqrt{2 \Phi_{n}^{\prime}(x)} \sqrt{2 g_{n}(x)} \\
= & \frac{1}{2 \beta}\left(\frac{1}{\sqrt[3]{g_{n}(x)}}+\frac{\sqrt{\Phi_{n}^{\prime}(x)}}{\sqrt{g_{n}(x)}}\right)<\frac{1+\sqrt[6]{2}}{2 \beta \sqrt[3]{g_{n}(x)}} .
\end{aligned}
$$

Считая, что $x \in[\alpha-2 \beta, \alpha+2 \beta]$, полученное неравенство можно записать в виде

$$
\left|\varphi(x)-D_{4 n-4}(x, f)\right|<\frac{6}{\sqrt[3]{g_{n}(x)}} \frac{\beta}{(x-\alpha)^{2}+\beta^{2}} .
$$

Если же $x \notin[\alpha-2 \beta, \alpha+2 \beta]$, то $\varphi(x)=0$ и

$$
\begin{aligned}
\left|\varphi(x)-D_{4 n-4}(x, f)\right| & \leqslant \frac{1}{2 g_{n}(x)} \sum_{k=1}^{2 n-1}\left|\varphi\left(x_{k}\right)\right| \frac{\sin ^{4}\left[\Phi_{n}(x)-\Phi_{n}\left(x_{k}\right)\right]}{\left(x-x_{k}\right)^{4} \Phi_{n}^{\prime}\left(x_{k}\right)} \\
& \leqslant \frac{1}{2 g_{n}(x)} \sum_{k=1}^{2 n-1} \frac{1}{2\left(x-x_{k}\right)^{4} \Phi_{n}^{\prime}\left(x_{k}\right)} \\
& \leqslant \frac{1}{4 g_{n}(x)} \frac{25}{\left((x-\alpha)^{2}+\beta^{2}\right)^{2}} \quad \sum_{\alpha-\beta \leqslant x_{k} \leqslant \alpha+\beta} \frac{1}{\Phi_{n}^{\prime}\left(x_{k}\right)} \\
& \leqslant \frac{1}{g_{n}(x)} \frac{75}{2 \pi} \frac{\beta}{\left((x-\alpha)^{2}+\beta^{2}\right)^{2}} \leqslant \frac{16 \beta}{\left((x-\alpha)^{2}+\beta^{2}\right)^{2}} \frac{1}{g_{n}(x)} .
\end{aligned}
$$

Из оценок (21) и (22) вытекает, что при всех $x \in \mathbb{R}$ вьполняется неравенство (20).

Будем рассматривать также линейные комбинации простых функций $\varphi_{k}(x)$ с неотрицательными коэффициентами $\lambda_{k}$

$$
f(x)=\lambda_{1} \varphi_{1}(x)+\lambda_{2} \varphi_{2}(x)+\cdots+\lambda_{n} \varphi_{n}(x)
$$

и приближать их сумматорными рациональными операторами $D_{4 m-4}(x, f)$, причем параметры $z_{k}$, по которым строится оператор (5), выбираются в соответствии с опорньми отрезками функций $\varphi_{k}(x)$, а их кратности определенным образом связываются с весовыми коэффициентами. 
Teорема 2. Если $f(x)=\sum_{k=1}^{n} \lambda_{k} \varphi_{k}(x)$, где $\lambda_{k} \geqslant 0 u\left\{\varphi_{k}(x)\right\}-$ простые функиии, то существует такой набор параметров $z_{1}, z_{2}, \ldots, z_{m}, m \leqslant 2 n$, ито для определяемого ими сумматорного оператора $D_{4 m-4}$ выполняется неравенство

$$
\left|f(x)-D_{4 m-4}(x, f)\right| \leqslant \frac{C}{n} \sum_{k=1}^{n} \lambda_{k}, \quad x \in \mathbb{R} .
$$

ДокАЗАТЕЛЬСтво. Пусть $\sum_{k=1}^{n}=\omega_{n},\left[\alpha_{k}-\beta_{k}, \alpha_{k}+\beta_{k}\right], \alpha_{k} \in \mathbb{R}, \beta_{k}>0,-$ опорный отрезок простой функции $\varphi_{k}(x)$. Оператор $D_{4 m-4}$ строим по системе параметров

$$
z_{k_{j}}=\alpha_{k}+i \beta_{k}, \quad k=1, \ldots, n, \quad j=1,2, \ldots,\left[n \frac{\lambda_{k}}{\omega_{n}}\right]+1 .
$$

Количество этих параметров

$$
m=\sum_{k=1}^{n} \sum_{j=1}^{\left[n \lambda_{k} / \omega_{n}\right]+1} 1=\sum_{k=1}^{n}\left(\left[n \frac{\lambda_{k}}{\omega_{n}}\right]+1\right) \leqslant \sum_{k=1}^{n}\left(n \frac{\lambda_{k}}{\omega_{n}}+1\right)=\frac{n}{\omega_{n}} \omega_{n}+n=2 n .
$$

В силу линейности оператора $D_{4 m-4}$ и леммы 4 для $x \in \mathbb{R}$ будем иметь

$$
\begin{aligned}
\left|f(x)-D_{4 m-4}(x, f)\right| \leqslant & \frac{6}{\sqrt[3]{g_{m}(x)}} \sum_{k=1}^{n} \lambda_{k} \frac{\beta_{k}}{\left(x-\alpha_{k}\right)^{2}+\beta_{k}^{2}} \\
& +\frac{16}{g_{m}(x)} \sum_{k=1}^{n} \lambda_{k} \frac{\beta_{k}}{\left(\left(x-\alpha_{k}\right)^{2}+\beta_{k}^{2}\right)^{2}} .
\end{aligned}
$$

Воспользовавшись леммой (3), можно утверждать, что, во-первых,

$$
\begin{aligned}
\sqrt[3]{g_{m}(x)} & \geqslant \frac{1}{\sqrt[3]{2}} \sum_{k=1}^{n}\left(\sum_{j=1}^{\left[n \lambda_{k} / \omega_{n}\right]+1} \frac{\beta_{k}}{\left(x-\alpha_{k}\right)^{2}+\beta_{k}^{2}}\right) \\
& =\frac{1}{\sqrt[3]{2}} \sum_{k=1}^{n}\left(\left[n \frac{\lambda_{k}}{\omega_{n}}\right]+1\right) \frac{\beta_{k}}{\left(x-\alpha_{k}\right)^{2}+\beta_{k}^{2}} \geqslant \frac{1}{\sqrt[3]{2}} \sum_{k=1}^{n} \frac{n \lambda_{k}}{\omega_{n}} \frac{\beta_{k}}{\left(x-\alpha_{k}\right)^{2}+\beta_{k}^{2}} \\
& =\frac{1}{\sqrt[3]{2}} \sum_{k=1}^{n} \frac{\lambda_{k} \beta_{k}}{\left(x-\alpha_{k}\right)^{2}+\beta_{k}^{2}} \frac{n}{\omega_{n}}
\end{aligned}
$$

и, во-вторых,

$$
g_{m}(x) \geqslant \frac{n}{\omega_{n}} \frac{1}{2} \sum_{k=1}^{n} \lambda_{k} \frac{\beta_{k}}{\left(\left(x-\alpha_{k}\right)^{2}+\beta_{k}^{2}\right)^{2}} .
$$

С помощью (25) и (26) из неравенства (24) найдем

$$
\left|f(x)-D_{4 m-4}(x, f)\right| \leqslant \frac{6 \sqrt[3]{2} \omega_{n}}{n}+\frac{32 \omega_{n}}{n}=\frac{C \omega_{n}}{n} .
$$

Теорема 2 доказана.

Заметим, что аппроксимация линейных комбинаций простых функций интегральными рациональными операторами и ее приложения к нахождению порядковых оценок для наилучших рациональных приближений исследованы А. А. Пекарским в [2]. 
ТЕОРема 3. Если $f(x) \in C(\mathbb{R})$ является четной, невозрастающей и выпуклой вниз на $(0, \infty), u \lim _{x \rightarrow \infty} f(x)=0$, то существует такой набор параметров $z_{1}, z_{2}, \ldots, z_{m}, m \leqslant 2 n$, что для определяемого ими сумматорного оператора верна оценка

$$
\left\|f(x)-D_{4 m-4}(x, f)\right\|_{C(\mathbb{R})}=O\left(\frac{1}{n}\right) .
$$

ДокАЗАТЕЛЬСтво. Пусть $\left\{x_{k}\right\}_{k=1}^{n}-$ абсциссы тех точек, для которых $f\left(x_{k}\right)=$ $(f(0) / n) k, k=1, \ldots, n$. Возьмем четную ломаную $\lambda(x)$ с вершинами в точках $\left(x_{k}, f\left(x_{k}\right)-f(0) / n\right), k=1, \ldots, n$, и в точках $\left(-x_{k}, f\left(x_{k}\right)-f(0) / n\right)$. Считаем, что $\lambda(x)=0$, если $x \in \mathbb{R} \backslash\left(-x_{1}, x_{1}\right)$. Очевидно, что вьполняется неравенство

$$
\|f(x)-\lambda(x)\| \leqslant \frac{f(0)}{n} .
$$

Ломаная $\lambda(x)$ представима в виде линейной комбинации

$$
\sum_{k=1}^{n-1} \lambda_{k} \varphi_{k}(x), \quad \lambda_{k}>0, \quad \sum_{k=1}^{n-1} \lambda_{k} \leqslant 2 f(0),
$$

простых треугольных функций $\varphi_{k}(x)=\frac{1}{2} \max \left\{0,1-|x| / x_{k}\right\}$ и, следовательно, по теореме 2 существует сумматорньй оператор $D_{4 m-4}, m \leqslant 2 n-2$, для которого вьполнено неравенство

$$
\left\|\lambda(x)-D_{4 m-4}(x, \lambda)\right\| \leqslant \frac{C_{1}}{n} f(0) .
$$

Тогда для уклонения значения этого оператора от функции $f(x)$ с учетом (28) и (29) будем иметь

$$
\begin{aligned}
\left\|f(x)-D_{4 m-4}(x, f)\right\|_{C(\mathbb{R})} \leqslant & \|f(x)-\lambda(x)\|_{C(\mathbb{R})}+\left\|\lambda(x)-D_{4 m-4}(x, \lambda)\right\|_{C(\mathbb{R})} \\
& +\left\|D_{4 m-4}(x, \lambda)-D_{4 m-4}(x, f)\right\|_{C(\mathbb{R})} \\
\leqslant & \frac{f(0)}{n}+\frac{C_{1}}{n} f(0)+\left\|D_{4 m-4}(x, \lambda-f)\right\|_{C(\mathbb{R})}=O\left(\frac{1}{n}\right)
\end{aligned}
$$

и соотношение (27) установлено.

Из теоремы 3 в качестве следствия вытекает известный результат (см. [7]-[9]): если $f(x) \in C[a, b]$ и выпуклая, то для наилучшего рачионального приближения верна оценка

$$
R_{n}(f, C[a, b])=O\left(\frac{1}{n}\right) .
$$

При интегрировании сумматорных рациональных операторов вида (5) возникают квадратурные формулы для вычисления несобственных интегралов. Если $f(x) \in C(\mathbb{R})$ и существует обший предел $\lim _{x \rightarrow \pm \infty} f(x)$, то справедлива квадратурная формула

$$
\int_{-\infty}^{\infty} \frac{f(x)}{1+x^{2}} d x=\pi f(\infty)+\pi \sum_{k=1}^{n} \frac{f\left(x_{k}\right)}{\left(1+x_{k}^{2}\right) \Phi_{n+1}^{\prime}\left(x_{k}\right)}+\rho_{n}(f),
$$


где $\left\{x_{k}\right\}_{k=1}^{n}$ суть нули синус-дроби Бернштейна $\sin \Phi_{n+1}(x)$,

$$
\Phi_{n+1}(x)=\sum_{k=0}^{n} \arg \left(z_{k}-x\right)
$$

$z_{0}=i$ Квадратурная формула (30) является точной на рациональных функциях

$$
r_{2 n}(x)=\frac{p_{2 n}(x)}{\prod_{k=1}^{n}\left(x-z_{k}\right)\left(x-\bar{z}_{k}\right)}
$$

т.е. для таких функций остаток квадратурной формулы $\rho_{n}\left(r_{2 n}\right)=0$.

В частном случае, если $z_{k}=i$,

$$
\Phi_{n+1}(x)=(n+1) \arccos \frac{-x}{\sqrt{x^{2}+1}}
$$

равенство (30) приводится к соотношению

$$
\int_{-\infty}^{\infty} \frac{f(x)}{1+x^{2}} d x=\pi f(\infty)+\frac{\pi}{n+1} \sum_{k=1}^{n} f\left(x_{k}\right)+\rho_{n}(f), \quad x_{k}=\operatorname{ctg} \frac{\pi k}{n+1}, \quad k=1, \ldots, n
$$

В заключение заметим, что основные результаты данной статьи были анонсированы в $[10]$.

\section{СПИСОК ЦИТИРОВАННОЙ ЛИТЕРАТУРЫ}

[1] Русак В. Н. Рациональные функции как аппарат приближения. Минск: Изд-во Белорус. ун-та, 1979.

[2] Пекарский А. А. Чебышевские рациональные приближения в круге, на окружности и на отрезке // Матем. сб. 1987. Т. 133. № 1. С. 86-102.

[3] Petrushev P. P., Popov V.A. Rational Approximation of Real Functions. Cambridge: Cambridge Univ. Press, 1987.

[4] Lorentz G.G., Golitschek M. V., Makovoz Y. J. Constructive Approximation. New York, 1996.

[5] Ровба Е. А. Интерполяционные рациональные операторы типа Фейера и Валле-Пуссена // Матем. заметки. 1993. Т. 53. № 2. С. 114-121.

[6] Ровба Е. А. Сумматорные рациональные операторы типа Джексона // Матем. заметки. 1997. T. 61. № 2. C. 270-277.

[7] Попов В. А., Петрушев П. П. Точный порядок наилучшего равномерного приближения вьпуклых функций рациональными функциями // Матем. сб. 1977. Т. 103. № 2. С. 285-292.

[8] Пекарский А. А. Рациональные приближения абсолютно непрерывных функций с производной из пространства Орлича // Матем. сб. 1982. Т. 117. № 1. С. 114-130.

[9] Буланов А. П. О порядке приближения выпуклых функций рациональными функциями // Изв. АН СССР. Сер. матем. 1969. Т. 33. № 5. С. 1132-1148.

[10] Русак В. Н., Рыбаченко И. В. Об одном методе приближения рациональными функциями // Докл. НАН Беларуси. 2002. Т. 4. № 5. С. 14-16. 